\title{
Naturwissenschaftliche
}

\section{Kompetenz in der Grundschule: Effekte von Familienmerkmalen und Klassenkomposition}

\author{
Jana Kähler @i), Inga Hahn und Olaf Köller \\ IPN Leibniz-Institut für die Pädagogik der Naturwissenschaften und Mathematik, Kiel, Deutschland
}

\begin{abstract}
Zusammenfassung: Im vorliegenden Beitrag wurden anhand der Daten von 6,492 Schülerinnen und Schülern der Startkohorte 2 des Nationalen Bildungspanels (NEPS) die Entwicklung der naturwissenschaftlichen Kompetenz von der 1. bis zur 3. Klasse sowie mögliche Effekte der Klassenkomposition unter Berücksichtigung relevanter Familienmerkmale untersucht. Die Analysen belegen im Mittel einen Zuwachs der naturwissenschaftlichen Kompetenz über zwei Jahre mit einer Effektstärke von $d=1.36$. Die Mehrebenenanalysen zeigen darüber hinaus, dass die Ausgangskompetenz in der 1. Klasse (standardisiertes Regressionsgewicht $\beta=.53$ ) der stärkste Prädiktor für die naturwissenschaftliche Kompetenz in der 3. Klasse ist. Des Weiteren zeigen sich für die Anzahl an Büchern ( $\beta=.05$ ), den sozioökonomischen Status ( $\beta=.06$ ) und die Bildung der Eltern ( $\beta=.09$ ) signifikante Effekte. Auf Klassenebene erweist sich das Ausgangsniveau in der 1. Klasse $(\beta=.46)$ als mit Abstand stärkster Prädiktor. Zusätzlich kann ein signifikanter Effekt der sozialen Zusammensetzung der Klasse $(\beta=-.17)$ nachgewiesen werden: Schülerinnen und Schüler aus Klassen mit einem höheren Anteil an Kindern mit niedrigem sozioökonomischen Status erreichen einen geringeren Kompetenzzuwachs als Kinder aus Klassen mit einer niedrigeren Anzahl an sozial benachteiligten Kindern. Auch die Anzahl an Stunden von Heimat- und Sachunterricht in den Klassen $(\beta=.11$ ) hat einen signifikanten Effekt. Demnach trägt mehr naturwissenschaftlicher Unterricht zu einem höheren Wachstum naturwissenschaftlicher Kompetenz bei. Abschließend zeigen Mediationsanalysen signifikant indirekte Effekte über die naturwissenschaftliche Kompetenz in der 1. Klasse. Sozial- und migrationsbedingte Disparitäten ergeben sich demnach bereits in der 1. Klasse und werden über die anfängliche naturwissenschaftliche Kompetenz auf die Kompetenz in der 3. Klasse vermittelt.
\end{abstract}

Schlüsselwörter: Naturwissenschaftliche Kompetenz, Familienmerkmale, Klassenkomposition, Mehrebenenanalysen, Mediationsanalyse

Scientific literacy in primary school: effects of family characteristics and class composition

\begin{abstract}
Using data of 6,492 students from Starting Cohort 2 of the National Educational Panel Study (NEPS), this article examines the development of scientific literacy from Grade 1 to Grade 3. Furthermore, the effects of family characteristics and class composition on the change in scientific literacy are analysed. The analyses reveal an average increase in scientific literacy over two years with an effect size of $d=1.36$. In multilevel analyses, the initial scientific literacy in Grade 1 (standardised regression weight $\beta=.53$ ) is the best predictor of scientific literacy in Grade 3. Additionally, the number of books $(\beta=.05)$, the socioeconomic status $(\beta=.06)$, and the educational level of the parents $(\beta=.09)$ show significant effects on the development of scientific literacy. At class level, class-average scientific literacy in Grade 1 ( $\beta=.46)$ proved to be by far the strongest predictor. Moreover, the social composition of the class ( $\beta=-.17)$ has a significant effect on the change in scientific literacy: Students from classes with a higher number of children with low socioeconomic status show a smaller increase in scientific literacy than students from classes with a lower number of children with low socioeconomic status. The number of science lessons $(\beta=.11)$ also effects the scientific literacy. According to this, more science lessons contributed to higher growth in scientific literacy. Finally, a mediation analysis shows significant indirect effects on scientific literacy in Grade 3 through the scientific literacy in Grade 1. This means, disparities related to the social and migration background already existed in Grade 1 of primary school, and they are conveyed via the initial scientific literacy to scientific literacy in Grade 3.
\end{abstract}

Keywords: Scientific literacy, family features, composition effects, multilevel analysis, mediation analysis

\section{Einleitung}

In Zeiten der Corona-Pandemie, des Klimawandels oder des Bestrebens nachhaltiger zu leben nimmt die Bedeutsamkeit naturwissenschaftlicher Kompetenzen immer weiter zu. Um sich diesen gesellschaftlichen Herausforderungen zu stellen, bedarf es wissenschaftlichen und technologischen Fortschritts, der jedoch nur mit ausreichender naturwissenschaftlicher Kompetenz (NK) im Sinne einer Scientific Literacy in der Bevölkerung erreicht werden 
kann. Dabei ist sowohl ein Grundverständnis naturwissenschaftlicher Denk- und Arbeitsweisen als auch ein grundlegendes naturwissenschaftliches Wissen vonnöten, um Informationen bewerten und verantwortungsvoll handeln zu können (Schiepe-Tiska et al., 2017). Erst dann ermöglicht NK einen adäquaten Umgang mit den alltäglichen Herausforderungen und Problemen in unserer Umwelt (OECD, 2018). Da NK in diesem Sinne nicht nur individuell bedeutsam ist, sondern auch zum Wirtschaftswachstum eines Landes beiträgt (Hanushek \& Wößmann, 2015), ist es umso wichtiger, frühzeitig eine solide Basis für einen gut ausgebildeten Nachwuchs in den Natur- und Ingenieurswissenschaften zu schaffen (OECD, 2018).

Obwohl aufgrund der wachsenden Herausforderungen NK immer mehr an Relevanz gewinnt, gibt es in Deutschland bisher nur sehr wenige Untersuchungen zur Entwicklung der NK und möglicher Einflussgrößen. Befunde, die Erkenntnisse über die NK von Kindern und Jugendlichen liefern, kamen in den letzten Jahren vornehmlich aus dem Bereich der Schulleistungsstudien wie der Trends in International Mathematics and Science Study (TIMSS, Wendt et al., 2016) oder dem Programme for International Student Assessment (PISA, OECD, 2006). Diese wiederkehrenden Querschnittstudien zeigten sowohl für den Primar- als auch den Sekundarbereich deutliche sozial- und migrationsbedingte Disparitäten der Schülerinnen und Schüler (SuS) im Hinblick auf ihre NK. Studien wie TIMSS oder PISA ermöglichen aufgrund ihres Querschnittsdesigns jedoch keine Untersuchung der Entwicklung der NK und möglicher Effekte von häuslichen und institutionellen Merkmalen auf diese Entwicklung. Eine Studie, mit deren Hilfe diese Forschungslücke jetzt verkleinert werden kann, ist das Nationale Bildungspanel (National Educational Panel Study, NEPS). Hier wird die NK (neben anderen Kompetenzdomänen) in verschiedenen Startkohorten für verschiedene Altersstufen längsschnittlich untersucht (Blossfeld, Roßbach \& Maurice, 2011). Die NEPS-Daten der Startkohorte 2 sollen in diesem Beitrag genutzt werden, um zu untersuchen, wie sich die NK in der Grundschule von der 1 . bis zur 3. Klasse entwickelt und welche individuellen und klassenbezogenen Merkmale relevante Prädiktoren für die Veränderung darstellen.

\section{Naturwissenschaftliche Kompetenz im NEPS}

Die Rahmenkonzeption der Naturwissenschaftstests im NEPS basiert auf dem Konzept der Scientific Literacy (Bybee, 1997), an dem sich z.B. auch die naturwissenschaftliche Rahmenkonzeption der PISA-Studie orientiert
(OECD, 2006). Dabei wird NK als Teil der Grundbildung verstanden, die Menschen dazu befähigt, sich mit den wachsenden naturwissenschaftlichen und technologischen Anforderungen in der Gesellschaft auseinanderzusetzen (OECD, 2006). Sie ermöglicht somit die Bewältigung alltäglicher Situationen und Probleme im Bereich der Naturwissenschaften. Darauf aufbauend wird NK in zwei Komponenten unterteilt: naturwissenschaftliches Wissen (Knowledge of Science) und Wissen über die Naturwissenschaften (Knowledge about Science). Diese Unterteilung findet nicht nur bei der Aufgabenerstellung in PISA (OECD, 2006) oder NEPS (siehe Elektronisches Supplement [ESM] 1; Hahn et al., 2013) Anwendung, sondern ist auch Teil verschiedener Standards im Grundschulbereich (Gesellschaft für Didaktik des Sachunterrichts, 2002).

Das naturwissenschaftliche Wissen bezieht sich auf inhaltsbezogene Komponenten, also auf naturwissenschaftliche Konzepte und Fakten. Kinder verfügen bereits in ihren ersten Lebensjahren über anschlussfähige naturwissenschaftliche Konzepte (Carey, 2009), die bei der Interpretation verschiedenster Phänomene unserer Welt herangezogen werden (Pollmeier et al., 2009). Diese anfänglichen, meist naiven Konzepte und Vorstellungen werden dabei mithilfe von Alltagserfahrungen aufgebaut und verankert (Jonen, Möller \& Hardy, 2003). Jedoch sind diese naiven Vorstellungen nur selten mit den zu lernenden, wissenschaftlich korrekten Konzepten und Erklärungsmodellen vereinbar (Duit \& Häußler, 1997; Duit, 1999). D.h. Kinder verfügen bereits vor dem Besuch der Grundschule über eine Reihe von naturwissenschaftlichen Fehlvorstellungen. Daher bedarf es in der Grundschule in aller erster Linie einer grundlegenden Umstrukturierung und Korrektur bestehender Fehlkonzepte hin zu wissenschaftlich verlässlichen Konzepten, um damit anschlussfähiges Lernen zu ermöglichen (Hardy et al., 2010; Pollmeier et al., 2009). In der Grundschule liegt der Fokus vor allem auf der Vermittlung naturwissenschaftlicher Konzepte in den Bereichen Physik (z.B. Magnetismus oder Auftriebs- und Gewichtskraft), Biologie (z.B. Strukturen und Funktionen in der belebten Natur) und Chemie (z.B. Stoffe, Energieumwandlung oder chemische Reaktionen; Hardy et al., 2010). Daher werden diese drei Bereiche auch bei der Erfassung NK bei PISA, den Bildungsstandards und dem NEPS berücksichtigt (Hahn et al., 2013). Für eine NEPS-Beispielaufgabe des Naturwissenschaftstests der 3. Klasse siehe ESM 2.

Das Wissen über die Naturwissenschaften bezieht sich auf das Verständnis naturwissenschaftlicher Denk- und Arbeitsweisen und wird im NEPS als prozessbezogene Komponente aufgefasst. Es beinhaltet das Formulieren von Hypothesen, das Testen der Hypothesen mittels entsprechender Experimente und die abschließende Interpretation der Ergebnisse dieser Experimente (Zimmer- 
man, 2007). Bereits im Grundschulalter zeigt sich, dass Kinder in der Lage sind, einfache Hypothesen zu formulieren und diese mithilfe eines passenden Experiments zu überprüfen (Sodian \& Mayer, 2013). Darüber hinaus konnte gezeigt werden, dass wissenschaftstheoretisch orientierter Unterricht das Wissen über die Naturwissenschaften von Grundschulkindern signifikant verbessern kann und dass dieser Unterricht außerdem die Fähigkeit zur Produktion kontrollierter Experimente positiv beeinflusst (Sodian, Jonen, Thoermer \& Kircher, 2006). Dabei lassen sich auch im Bereich des Wissens über die Naturwissenschaften naive Vorstellungen finden, die erst durch grundlegende Umstrukturierung korrigiert werden können (Hardy et al., 2010). Für eine NEPS-Beispielaufgabe des Naturwissenschaftstests der 3. Klasse siehe ESM 3.

\section{Befunde zur Entwicklung der naturwissenschaftlichen Kompetenz}

Im Folgenden sollen der bisherige Forschungsstand zur NK und ihrer Entwicklung präsentiert werden. Bisher gibt es nur wenige groß angelegte Studien für den Bereich der frühen Kindheit, sodass für Annahmen über die Entwicklung von NK in diesem Bereich auch auf Erkenntnisse aus der Sekundarstufe I zurückgegriffen werden muss. Dies ermöglicht jedoch auch einen Vergleich der Ergebnisse dieser Studie bzw. anderer Studien in der Grundschule mit bereits gefundenen Wachstumsfortschritten im Sekundarbereich.

Morgan, Farkas, Hillemeier und Maczuga (2016) untersuchten mithilfe von Daten der Early Childhood Longitudinal Study (ECLS-K) die Entwicklung der NK (bzw. früher Vorläuferfähigkeiten) von 7,757 Kindern vom Kindergarten bis zur 8. Klasse. Die Daten zeigten dabei besonders vom Kindergarten bis zur 1. Klasse ein starkes Kompetenzwachstum der Kinder $(d=1.59)$. Das Wachstum der NK ab der 3. Klasse fiel dagegen etwas geringer aus (Klasse 3 bis 5 : $d=0.88$; Klasse 5 bis 8: $d=1.14$ ). Die Autoren konnten zusätzlich bereits im Kindergarten große Kompetenzunterschiede im Bereich der Natur- und Sozialwissenschaften nachweisen. Vor allem Kinder mit sprachbezogenen und sozialen Nachteilen zeigten hier niedrigere Kompetenzen.

Auch anhand der Daten des NEPS wurde die Entwicklung NK in der frühen Kindheit untersucht (Kähler, Hahn \& Köller, 2020). Wachstumskurvenmodelle konnten mit den Daten der Startkohorte $2(N=2,937)$ nachweisen, dass die NK vom Kindergarten über die Zeit in der Grundschule relativ stabil wächst (Kindergarten bis Klasse 1: $d=1.23$; Klasse 1 bis 3: $d=1.25)$. Darüber hinaus zeigte sich, dass sich die Kinder bereits im Kindergarten signifikant in ihrer NK unterschieden und dass diese Unterschiede über die Grundschulzeit bestehen blieben ${ }^{1}$. Analysen zu den Effekten familiärer und institutioneller Merkmale in der frühen Kindheit ergaben, dass sich diese Disparitäten zum Teil auf Unterschiede in den sprachlichen Kompetenzen, zum Teil aber auch auf sozial- und migrationsbedingte Unterschiede zurückführen ließen (Hahn \& Schöps, 2019; Kähler, Hahn, Ihme \& Köller, 2020). So zeigte sich u.a., dass Kinder mit Migrationshintergrund (gemessen über das Geburtsland der Eltern) eine deutlich geringere NK aufwiesen als ihre Peers ohne Migrationshintergrund (Hahn \& Schöps, 2019).

Der Effekt des Migrationshintergrundes wurde ebenfalls in der TIMSS-Studie anhand von 3,948 Kindern der 4. Klasse in Deutschland untersucht (Wendt et al., 2016). Auch hier konnten sozial- und migrationsbedingte Disparitäten festgestellt werden. Kinder mit Migrationshintergrund, aus Arbeiterfamilien oder armutsgefährdete Kinder zeigten deutlich niedrigere Leistungen in den Naturwissenschaften im Vergleich zu ihren Peers ohne soziale Benachteiligung. Darüber hinaus untersuchte die TIMSS-Studie der 1990er Jahre die Leistungsfortschritte der NK deutscher SuS von der 7. zur 8. Klasse (Baumert et al., 1997). Dabei fielen die Fortschritte im internationalen Vergleich deutlich geringer aus $(d=0.33)$.

Den Zuwachs der NK von der 7. zur 8. Klasse untersuchten auch Ivanov und Nikolova (2010) anhand der Daten der Studie Kompetenzen und Einstellungen von Schülerinnen und Schülern am Ende der Jahrgangsstufe 8 (KESS). Der Naturwissenschaftstest der KESS-Studie basierte dabei weitestgehend auf den curricular validen Testaufgaben der TIMSS-Studie und wurde bei 4,884 SuS aus Hamburg eingesetzt. Es zeigte sich ein deutlich höherer Kompetenzzuwachs $(d=0.81)$ als bei der TIMSSStudie (vgl. Baumert et al., 1997).

Ein wiederum mit den TIMSS-Daten vergleichbares Ergebnis lieferte die nationale Erweiterung der PISA Studie 2003. In einer Follow-up-Untersuchung wurde anhand curricular verankerter Testaufgaben die Entwicklung der NK von der 9. zur 10. Klasse ermittelt. Dabei ergab sich ein Kompetenzzuwachs von $d=0.24$ Standardabweichungen (Walter, Senkbeil, Rost, Carstensen \& Prenzel, 2006). Im Gegensatz dazu fanden Schiepe-Tiska et al. (2017) in ihrer Untersuchung des PISA-Längsschnitts 2012/2013 bei der Analyse der internationalen Testaufgaben keine Veränderungen in der NK von der 9. zur 10. Klasse $(d=0.02)$.

Insgesamt zeigt sich, dass die Kompetenzzuwächse in den ersten Jahren der Grundschule deutlich höher ausfallen als die Entwicklung in der Sekundarstufe I. Damit lassen sich vergleichbare Verhältnisse aufzeigen wie im 
Bereich der Lese- und Mathematikleistungen (Köller \& Baumert, 2018). Es lassen sich somit auch für die NK nicht-lineare Lernzuwächse vermuten, d.h. die Leistungszuwächse nehmen in den höheren Klassen immer weiter ab (Köller \& Baumert, 2018). Mit dieser Untersuchung soll daher geprüft werden, ob sich die Entwicklung der NK in der Grundschule anhand der NEPS-Daten ebenfalls in die bestehenden Ergebnisse einordnen lässt.

\section{Determinanten der Entwicklung naturwissenschaftlicher Kompetenz}

\section{Effekte struktureller Familienmerkmale}

Zahlreiche nationale und internationale Large-Scale-Studien konnten nachweisen, dass Merkmale des Elternhauses eine wichtige Grundlage für die kognitive Entwicklung von der frühen Kindheit bis ins Jugendalter darstellen (u.a. OECD, 2006; Wendt et al., 2016).

Insbesondere der sozioökonomische Status (SES) einer Familie erwies sich in umfassenden Studien als stabiler Prädiktor kognitiver Kompetenzen. Mit steigendem SES fielen u.a. auch die mathematischen und naturwissenschaftlichen Kompetenzen von Kindern und Jugendlichen höher aus (Ivanov \& Nikolova, 2010; Morgan et al., 2016; Opdenakker, van Damme, Fraine, van Landeghem \& Onghena, 2002). Zusätzlich zu dem über die berufliche Stellung der Eltern gemessenen SES einer Familie wurde als Prädiktor kindlicher Kompetenzen auch oft die Anzahl an Büchern zuhause (als Indikator für objektiviertes kulturelles Kapital) herangezogen. Untersuchungen im Kindergarten- und Grundschulbereich konnten hierzu einen positiven Effekt auf die mathematische und naturwissenschaftliche Kompetenz (Hahn \& Schöps, 2019; Wendt et al., 2016) sowie auf die Lesekompetenz (Ditton \& Krüsken, 2006) nachweisen. Somit zeigten sozial benachteiligte Kinder (ob anhand eines niedrigeren SES oder einer geringeren Anzahl an Büchern) bereits im Kindergarten und der Grundschule ein geringeres Kompetenzniveau als ihre sozial privilegierten Peers. Des Weiteren zeigten Kinder, deren Eltern einen höheren Bildungsabschluss aufwiesen, auch höhere Kompetenzen in den Bereichen Mathematik, Lesen und Naturwissenschaften als Kinder mit niedrigerer elterlicher Bildung (Baumert et al., 2006; Ditton \& Krüsken, 2006; Ivanov \& Nikolova, 2010; Walter, 2008). Neben den sozialen Aspekten der Familie steht auch der Migrationshintergrund, gebildet über das Geburtsland der Eltern eines Kindes, immer wieder als Indikator ethnischer Bildungsunterschiede im Fokus. Studien konnten nachweisen, dass Kinder mit Migrationshintergrund nicht nur geringere Lesefähigkeiten in Deutsch (Niklas, Segerer, Schmiedeler \& Schneider, 2012; Schwippert, Wendt \& Tarelli, 2012), sondern auch gerin- gere mathematische und naturwissenschaftliche Kompetenzen als Kinder ohne Migrationshintergrund aufwiesen (Hahn \& Schöps, 2019; Wendt et al., 2016). Darüber hinaus dient der häusliche Sprachgebrauch einer Familie in vielen Studien als Prädiktor kindlicher Kompetenzen. Auch hier zeigte sich, dass Kinder mit einer anderen $\mathrm{Fa}-$ miliensprache als Deutsch eine geringere NK aufwiesen (Hahn \& Schöps, 2019; Ivanov \& Nikolova, 2010).

\section{Effekte von Kompositionsmerkmalen}

Sowohl nationale als auch internationale Untersuchungen $\mathrm{zu}$ Kompositionsmerkmalen konnten übereinstimmend feststellen, dass das Fähigkeitsniveau einer Klasse (oder Schule) auch bei simultaner Berücksichtigung anderer Kompositionsmerkmale den stärksten Prädiktor für verschiedene Kompetenzen von SuS darstellte (für Lesekompetenz: Baumert, Maaz, Stanat \& Watermann, 2009; Bellin, 2009; für mathematische Kompetenz: Nikolova, 2011; Opdenakker et al., 2002; für NK: Schiepe-Tiska et al., 2017; für eine zusammenfassende Darstellung siehe Dumont, Neumann, Maaz und Trautwein, 2013).

Für den mittleren SES einer Klasse (oder Schule) ergab sich dagegen ein weniger eindeutiges Bild. Einige Studien konnten einen Effekt auch nach Berücksichtigung des mittleren Fähigkeitsniveaus (und anderer Kompositionsmerkmale) für die Lesekompetenz (Baumert et al., 2006; Baumert et al., 2009) und die mathematische Kompetenz (Opdenakker et al., 2002) nachweisen, während andere Studien keinen eigenständigen Effekt des mittleren SES für die Lesekompetenz (Bellin, 2009; Nikolova, 2011), mathematische Kompetenz (Dumont, Neumann, Nagy, Becker \& Rose, 2013; Nikolova, 2011) und NK (Schiepe-Tiska et al., 2017) finden konnten. Diese Diversität in den Befunden konnte durch die Meta-Analyse von Van Ewijk und Sleegers (2010) untermauert werden: In den 30 untersuchten Studien zeigte sich, dass die Effekte stark von der Art der Messung des SES und der Modellauswahl abhingen. Zum einen ergab ein dichotom gemessener SES einen geringeren Effekt als ein Komposit aus mehreren SES-Dimensionen, zum anderen fielen die Effekte auf Schulebene deutlich geringer aus als auf Klassenebene. Van Ewijk und Sleegers (2010) kamen zu der Schlussfolgerung, dass unter Berücksichtigung all dieser Punkte der mittlere SES eine bedeutsame Determinante für die Kompetenzen von SuS darstellt.

Neben der sozialen Komposition einer Schülerschaft stand auch die ethnisch-kulturelle Zusammensetzung im Fokus vieler Studien. Dabei war entweder der Anteil an SuS mit Migrationshintergrund oder der Anteil an SuS mit nicht-deutscher Muttersprache Gegenstand der Untersuchungen. Hier zeigte sich fast einheitlich, dass unter Berücksichtigung des mittleren Fähigkeitsniveaus und der sozialen Zusammensetzung der eigenständige Effekt der ethnischen Zusammensetzung auf die sprachliche bzw. 
naturwissenschaftliche Kompetenz verschwand (Bellin, 2009; Ditton \& Krüsken, 2006; Walter, 2008). Das bedeutet, dass die Nachteile einer Klasse mit einem höheren Anteil an SuS mit Migrationshintergrund vollständig auf den mittleren SES und das mittlere Fähigkeitsniveau zurückgeführt werden können (Bellin, 2009).

Insgesamt wird durch die dargestellten Befunde deutlich, dass in Untersuchungen zur Entwicklung der NK sowohl familiäre als auch institutionelle Merkmale berücksichtigt werden müssen. Allerdings sind Untersuchungen von Kompositionseffekten auf die NK in der bisherigen Forschung im Grundschulbereich selten. Es stellt sich die Frage, ob die für die Sekundarstufe I gefundenen Kompositionseffekte bereits in gleicher Weise in der Grundschule nachweisbar sind und inwiefern sich weiterhin sozial- und migrationsbedingte Nachteile des familiären Hintergrunds zeigen.

\section{Fragestellungen}

Ziel der vorliegenden Arbeit ist die Untersuchung der Veränderung der NK von SuS in der Grundschule (von der 1. bis zur 3. Klasse) und der möglichen Einflussfaktoren auf die Veränderung. Es soll der Frage nachgegangen werden, inwiefern die Komposition einer Klasse im ersten Jahr der Grundschule unter Berücksichtigung von individuellen Familienmerkmalen einen zusätzlichen Effekt auf die Kompetenzentwicklung in der 3. Klasse der Grundschule hat. Als Kompositionsmerkmale gehen dabei soziale, migrationsbedingte, geschlechtsspezifische und leistungsbezogene Merkmale in die Analysen ein. Es ergeben sich folgende Fragestellungen:

1. Wie hoch ist der Zuwachs der NK in den NEPS-Naturwissenschaftstests von der 1. bis zur 3. Klasse?

2. Welche Merkmale der Familie haben einen signifikanten Effekt auf die Veränderung der NK von der 1. bis zur 3. Klasse (unter Berücksichtigung der Merkmale der Klassenkomposition)?

3. Welche Merkmale der Klassenkomposition haben einen signifikanten Effekt auf die Veränderung der NK von der 1. bis zur 3. Klasse (unter Berücksichtigung der Merkmale der Familie)?

4. Inwiefern ergeben sich indirekte Effekte der Familienund Kompositionsmerkmale über die NK in der 1. Klasse auf die NK in der 3. Klasse?

\section{Methode}

\section{Stichprobe}

Für die vorliegende Untersuchung wurden die Daten von 6,492 Kindern (51\% Mädchen) aus 1,018 Klassen von 374 Grundschulen der Startkohorte 2 des NEPS verwendet
(Blossfeld et al., 2011). Die Kompetenzmessungen fanden in der 1. und 3. Klasse der Grundschule statt. Von Februar bis Juni 2013 nahmen 6,492 Kinder an der Kompetenzmessung in der 1. Klasse teil (Alter: $M=7.08$ Jahre, $S D=$ 0.40 , Range $=5.42$ bis 8.92 Jahre). Bei der Kompetenzmessung in der 3. Klasse von November 2014 bis Januar 2015 nahmen noch 5,327 Kinder teil (Alter: $M=8.67$ Jahre, $S D=0.38$, Range $=7.00$ bis 10.58 Jahre). Für eine detaillierte Beschreibung der Stichprobe siehe ESM 4.

Da die Teilnahme freiwillig war, wurden keine kompletten Klassen getestet. Nur die Kinder, für die das Einverständnis der Eltern vorlag, durften an der Studie teilnehmen. Im Durchschnitt nahmen sechs SuS pro Klasse und drei Klassen pro Schule an der Studie teil.

\section{Instrumente und Variablen}

\section{Naturwissenschaftstests}

Die Erhebung der NK erfolgte mithilfe der NEPS-Naturwissenschaftstests für die 1. und 3. Klasse (Hahn et al., 2013; Kähler, 2019a, 2019b). Die Tests enthielten in der 1. Klasse 25 Items und in der 3 . Klasse 22 Items zu naturwissenschaftlichen Themen wie Tiere, Pflanzen oder Technik. Dabei kamen verschiedene Antwortformate zum Einsatz: ein einfaches Multiple-Choice-Format (vier Antworten, eine richtige) und ein komplexes Multiple-Choice-Format (vier Antworten, jeweils mit ja oder nein zu bewerten). Darüber hinaus enthielt der Test für die 3. Klasse noch zwei Aufgaben mit Kurztextantworten. Beide Kompetenzerhebungen wurden in Gruppentestungen durchgeführt, bei denen die Testleiterinnen und Testleiter die Items vorlasen. Im NEPS-Naturwissenschaftstest für die 1. Klasse waren die Antworten bildbasiert, d.h. die Kinder mussten ihre Antwort nicht formulieren, sondern nur das richtige Bild ankreuzen. Im NEPS-Naturwissenschaftstest für die 3. Klasse durften die Kinder die Fragen und Antworten mitlesen, mussten aber auch hier lediglich die Antworten ankreuzen. Für den Test in der 1. Klasse hatten die Kinder 30 Minuten Zeit, für den Test in der 3. Klasse 29 Minuten.

Auf Basis der Itemantworten wurden für beide Messzeitpunkte weighted maximum likelihood estimates (WLEs) als Fähigkeitsparameter berechnet. Dazu wurde ein eindimensionales Raschmodell in ConQuest 4.2.5 (Adams, Wu \& Wilson, 2015) angewendet. Die WLE-Reliabilität des Tests in der 1. Klasse lag bei $r=.73$ (Kähler, 2019a) und die des Tests in der 3. Klasse bei $r=.68$ (Kähler, 2019b). Da beide Tests unterschiedliche Aufgaben enthielten, die zur genauen Messung der NK in den Altersgruppen konstruiert wurden, musste ein Anchor-Group-Design verwendet werden, um die Fähigkeiten auf einer gemeinsamen, vergleichbaren Metrik abzubilden (siehe ESM 5). Um das Kompetenzniveau auf Klassenebene in den Mehrebenenanalysen 
zu berücksichtigen, wurde dies über die einzelnen WLEs der SuS einer Klasse aggregiert. Für weitere Informationen zu dem Linking der Tests siehe Kähler (2019b), für allgemeine Informationen zu der Linking Methode siehe Fischer, Rohm, Gnambs und Carstensen (2016).

\section{Familienmerkmale}

Mittels Fragebogen wurden die Eltern der teilnehmenden Kinder zu den familiären Merkmalen befragt (siehe ESM 4). Neben dem Geschlecht $(1=$ männlich, 2 = weiblich $)$ wurde die zuhause gesprochene Familiensprache $(1=$ Deutsch, 2 = andere Sprache) erfasst. Darüber hinaus wurde der Migrationshintergrund des Kindes ermittelt. Dieser definierte sich über das Geburtsland der Eltern $(1=$ kein Elternteil im Ausland geboren, 2 = ein Elternteil im Ausland geboren, $3=$ be de Eltern im Ausland geboren). Des Weiteren wurde die Anzahl der Bücher im Elternhaus $(1=0$ bis 10 Bücher bis 6 = mehr als 500 Bücher) als Maß für die Investitionsbereitschaft in kulturelles Kapital berücksichtigt. Der SES wurde mithilfe des International Socio-Economic Index of Occupational Status (ISEI; Ganzeboom, de Graaf \& Treiman, 1992) erfasst, welcher die sozioökonomische Stellung der Eltern über den Beruf ermittelt. Ein höherer Wert steht dabei für eine höhere berufliche Stellung. Darüber hinaus wurde auch das monatliche Netto-Einkommen der Eltern erhoben $(1=$ weniger als $1,000 €, 7=$ mehr als 6,000€). Das Bildungsniveau der Eltern wurde mit der International Standard Classification of Education (ISCED; Schroedter, Lechert \& Lüttinger, 2006) erfasst $(1=$ vorschulische Erziehung bis 6 = tertiäre Bildung). Sowohl für den SES als auch für die Bildung der Eltern wurde der jeweils höhere Wert verwendet, wenn Angaben beider Elternteile vorlagen.

\section{Klassenmerkmale}

Da keine ganzen Klassen erhoben wurden und im Mittel nur sechs SuS pro Klasse (Min = 1, Max = 21) getestet wurden, wurden die Kompositionsmerkmale einer Klasse nicht über die Individualangaben der einzelnen SuS einer Klasse aggregiert, sondern es wurde auf die Angaben der mittels Fragebogen befragten Lehrkräfte zurückgegriffen (siehe Befragungsinstrumente unter https://www.nepsdata.de/Datenzentrum/Daten-und-Dokumentation/ Startkohorte-Kindergarten/Dokumentation). Die Lehrkräfte machten bezogen auf die gesamte Klasse Angaben zum Geschlecht, SES (Familien aus eher niedrigen/mittleren/höheren sozialen Schichten), Migrationshintergrund (SuS selbst oder mindestens ein Elternteil im Ausland geboren) und zur Bildung der Eltern (mindestens ein Elternteil ein Studium abgeschlossen) der SuS. Des Weiteren wurden die Lehrkräfte auch zu der Anzahl an Unterrichtsstunden pro Woche $(1=$ unter $5 h, 2=5$ bis $6 h, 3=$ über $6 h$ ) in den Fächern Deutsch/Schriftspracherwerb, Mathematik und Heimat- und Sachunterricht befragt.
Diese gingen ebenfalls als Klassenmerkmale in die Analysen mit ein.

\section{Statistische Analysen und Umgang mit fehlenden Werten}

Zur Beantwortung der ersten Fragestellung werden zunächst deskriptive Ergebnisse zur Entwicklung der NK berichtet. Anschließend werden zur Beantwortung der zweiten bis vierten Fragestellung die Ergebnisse von Mehrebenen-Regressionsanalysen mit SuS auf Ebene 1 (Individualebene) und Klassen auf Ebene 2 (Kontextebene) in Mplus 7.4 (Muthén \& Muthén, 2012) präsentiert. Um den Zuwachs der NK untersuchen zu können, wurde die Kompetenz zum zweiten Messzeitpunkt (3. Klasse) als erstes durch die individuelle Kompetenz zum ersten Messzeitpunkt (1. Klasse) vorhergesagt. In den Regressionsmodellen wurden dann schrittweise weitere Prädiktoren der Individual- und Klassenebene aufgenommen, die den Zuwachs der NK vorhersagen sollten. Zu Beginn wurde ein Nullmodell zur Bestimmung der Intraklassenkorrelation spezifiziert. In Modell 1 wurde als erstes auf Ebene 1 die individuelle Ausgangskompetenz in der 1. Klasse aufgenommen. Modell 2 enthielt die individuellen Familienmerkmale auf Ebene 1. In Modell 3 wurde die mittlere Ausgangskompetenz der Klasse (1. Klasse) integriert, welche in Modell 4 um die Kompositionsmerkmale der Klassen erweitert wurde. In Modell 5 wurde dann als letztes die Anzahl an Unterrichtsstunden der Fächer Deutsch, Mathematik und Heimat- und Sachunterricht (HSU) aufgenommen. Modell 6 entspricht Modell 5, enthält aber zusätzlich die Mediation der Familien- und Kompositionsmerkmale über die NK der 1. Klasse auf die NK der 3. Klasse. In allen Analysemodellen wurden Stichprobengewichte für die Testpersonen verwendet, die das NEPS zur Verfügung stellt. Stichprobengewichte tragen dazu bei, Verzerrungen und andere Abweichungen zwischen der Stichprobe und der Referenzpopulation zu korrigieren und damit Verzerrungen in den Analyseergebnissen zu verhindern. Des Weiteren wurden die Variablen auf Individualebene am Gesamtmittelwert zentriert, um eine Interpretation der Kontexteffekte zu ermöglichen.

Um einer verzerrten Parameterschätzung aufgrund fehlender Werte (siehe Tabelle 1) entgegenzuwirken, wurde vor den Analysen eine multiple Imputation der fehlenden Daten ( $N=100$ Datensätze, 50 Iterationen) mithilfe des Programms RStudio V3.5.2 (RStudio Team, 2015) durchgeführt. Damit wurden keine Daten aufgrund von fehlenden Angaben aus den Analysen ausgeschlossen. Für die Imputation wurde das R-Paket mice verwendet, das die Mehrebenstruktur der Daten (SuS geschachtelt in Klassen) bei der Imputation berücksichtigt (Lüdtke, Robitzsch \& Grund, 2017). Eine abschließende Konvergenzdiagnostik in mice 
Tabelle 1. Deskriptive Statistiken der berücksichtigten Variablen

\begin{tabular}{|c|c|c|c|c|c|c|}
\hline Variablen & $N$ & Min & Max & $M$ & $S D$ & Missings \\
\hline \multicolumn{7}{|l|}{ Kind- und Familienmerkmale } \\
\hline Geschlecht (1 = männlich) & 6,492 & 1 & 2 & 1.51 & 0.50 & 0.00 \\
\hline Sprache zuhause (1 = deutsch) & 4,115 & 1 & 2 & 1.06 & 0.24 & 0.37 \\
\hline Migrationshintergrund (1 = kein) & 5,169 & 1 & 3 & 1.35 & 0.66 & 0.20 \\
\hline Anzahl der Bücher & 5,688 & 1 & 6 & 4.12 & 1.28 & 0.12 \\
\hline SES (HISEI) & 5,774 & 16 & 90 & 55.54 & 16.26 & 0.11 \\
\hline Bildung der Eltern (HISCED) & 5,842 & 1 & 6 & 4.63 & 1.32 & 0.10 \\
\hline Einkommen & 5,734 & 1 & 7 & 4.80 & 1.55 & 0.12 \\
\hline \multicolumn{7}{|l|}{ Klassenmerkmale } \\
\hline SuS pro Klasse & 5,702 & 1 & 5 & 2.94 & 0.80 & 0.12 \\
\hline SuS, männlich (\%) & 5,702 & 15 & 79 & 50.05 & 9.15 & 0.12 \\
\hline SuS, mit Migrationshintergrund (\%) & 5,219 & 0 & 100 & 26.93 & 23.53 & 0.20 \\
\hline SuS, mit niedrigem SES (\%) & 4,374 & 0 & 100 & 21.33 & 18.40 & 0.33 \\
\hline SuS, mit hohem SES (\%) & 4,323 & 0 & 100 & 19.39 & 18.10 & 0.33 \\
\hline SuS, Eltern mit Hochschulabschluss (\%) & 2,990 & 0 & 100 & 25.90 & 20.28 & 0.54 \\
\hline Unterrichtsstunden: Deutsch & 5,118 & 1 & 3 & 2.20 & 0.46 & 0.21 \\
\hline Unterrichtsstunden: Mathematik & 5,076 & 1 & 3 & 1.87 & 0.44 & 0.22 \\
\hline Unterrichtsstunden: HSU & 5,091 & 1 & 3 & 2.01 & 0.52 & 0.22 \\
\hline \multicolumn{7}{|l|}{ Kompetenzen } \\
\hline Naturwissen. Kompetenz 1. Klasse & 6,492 & -1.67 & 5.52 & 1.38 & 0.93 & 0.00 \\
\hline Naturwissen. Kompetenz 3. Klasse & 5,327 & -1.25 & 7.14 & 2.67 & 0.93 & 0.18 \\
\hline
\end{tabular}

Anmerkungen: N = 6,492; Min = Minimum, Max = Maximum, M = Mittelwert, SD = Standardabweichung, SES = sozioökonomischer Status, SuS = Schülerinnen und Schüler, HSU = Heimat- und Sachunterricht.

zeigte, dass der Imputationsalgorithmus stabil verlaufen ist und sich keine systematischen Verschiebungen im Imputationsverlauf ergeben haben. Für eine allgemeine Diskussion von Imputationsverfahren in Mehrebenenanalysen siehe Lüdtke et al. (2017).

Tabelle 1 zeigt die deskriptiven Statistiken der in die Analysen eingegangenen Variablen (vor der Imputation).

\section{Ergebnisse}

\section{Veränderung der naturwissenschaftlichen Kompetenz}

Zur Beantwortung der ersten Fragestellung wurde mithilfe der vorliegenden Mittelwerte und Standardabweichungen der NK auf Ebene der Schülerschaft die standardisierte
Effektgröße berechnet, um den Zuwachs für die NK zu quantifizieren (Hill, Bloom, Black \& Lipsey, 2008). Der Zuwachs betrug $d=1.36$ Standardabweichungen (CI [1.32, 1.40]). Damit zeigte sich ein deutliches Wachstum der NK von der 1. bis zur 3. Klasse.

\section{Effekte von Familien- und Klassen- merkmalen auf die Veränderung der naturwissenschaftlichen Kompetenz}

Zur Beantwortung der zweiten bis vierten Fragestellung wurden Mehrebenen-Regressionsanalysen durchgeführt. Ergebnisse der untersuchten Modelle finden sich in Form von standardisierten Regressionsgewichten in den Tabellen 2 und 3.

Zur Bestimmung der Verteilung der Varianz auf Individual- und Klassenebene in der 3. Klasse wurde als erstes 
Tabelle 2. Ergebnisse der Mehrebenenanalysen zur Vorhersage der naturwissenschaftlichen Kompetenz in Klasse 3

\begin{tabular}{|c|c|c|c|c|c|c|c|c|c|c|}
\hline \multirow{2}{*}{$\frac{\text { Variablen }}{\text { Ebene } 1}$} & \multicolumn{2}{|c|}{ Modell 1} & \multicolumn{2}{|c|}{ Modell 2} & \multicolumn{2}{|c|}{ Modell 3} & \multicolumn{2}{|c|}{ Modell 4} & \multicolumn{2}{|c|}{ Modell 5} \\
\hline & $\beta$ & $S E$ & $\beta$ & $S E$ & $\beta$ & $S E$ & $\beta$ & $S E$ & $\beta$ & $S E$ \\
\hline Naturwissen. Kompetenz 1.Klasse & $.61^{\star}$ & 0.01 & $.55^{\star}$ & 0.01 & $.53 *$ & 0.01 & $.52^{\star}$ & 0.01 & $.52 \star$ & 0.01 \\
\hline Geschlecht (1 = männlich) & & & $-.05 \star$ & 0.01 & $-.05^{\star}$ & 0.01 & $-.05 \star$ & 0.01 & $-.05^{\star}$ & 0.01 \\
\hline Sprache zuhause (1 = deutsch) & & & .01 & 0.01 & .01 & 0.02 & .01 & 0.02 & .01 & 0.02 \\
\hline Migrationshintergrund (1 = kein) & & & -.02 & 0.02 & -.02 & 0.02 & -.02 & 0.02 & -.02 & 0.02 \\
\hline Anzahl Bücher & & & $.04^{\star}$ & 0.02 & $.05^{\star}$ & 0.02 & $.04^{\star}$ & 0.02 & $.04^{*}$ & 0.02 \\
\hline SES (HISEI) & & & $.05^{\star}$ & 0.02 & $.05^{\star}$ & 0.02 & $.05^{\star}$ & 0.02 & $.05^{\star}$ & 0.02 \\
\hline Bildung der Eltern (HISCED) & & & $.09 *$ & 0.02 & $.09 *$ & 0.02 & $.08^{\star}$ & 0.02 & $.08 *$ & 0.02 \\
\hline Einkommen & & & $.03 *$ & 0.02 & $.03 *$ & 0.02 & .03 & 0.02 & .03 & 0.02 \\
\hline \multicolumn{11}{|l|}{ Ebene 2} \\
\hline Mittlere naturwissen. Kompetenz 1.Klasse & & & & & $.55^{\star}$ & 0.06 & $.48^{\star}$ & 0.06 & $.47 *$ & 0.07 \\
\hline SuS pro Klasse & & & & & & & -.03 & 0.05 & -.02 & 0.05 \\
\hline SuS, männlich (\%) & & & & & & & .02 & 0.05 & .02 & 0.05 \\
\hline SuS, mit Migrationshintergrund (\%) & & & & & & & -.01 & 0.07 & -.02 & 0.07 \\
\hline SuS, mit niedrigem SES (\%) & & & & & & & $-.17 \star$ & 0.08 & $-.17 \star$ & 0.08 \\
\hline SuS, mit hohem SES (\%) & & & & & & & .05 & 0.07 & .05 & 0.07 \\
\hline SuS, Eltern mit Hochschulabschluss (\%) & & & & & & & $.15^{\star}$ & 0.07 & .14 & 0.08 \\
\hline Unterrichtsstunden: Deutsch & & & & & & & & & .03 & 0.05 \\
\hline Unterrichtsstunden: Mathematik & & & & & & & & & .04 & 0.05 \\
\hline Unterrichtsstunden: HSU & & & & & & & & & $.11 *$ & 0.05 \\
\hline \multicolumn{11}{|l|}{ Varianzaufklärung } \\
\hline Ebene 1 & .38 & & .40 & & .38 & & .36 & & .36 & \\
\hline Ebene 2 & - & & - & & .30 & & .42 & & .44 & \\
\hline
\end{tabular}

Anmerkungen: $N=6,492 ; \beta=$ standardisierte Regressionsgewichte, $S E$ = Standardfehler, SES = sozioökonomischer Status, SuS = Schülerinnen und Schüler, $\mathrm{HSU}=$ Heimat - und Sachunterricht.

$* p<.05$.

ein Nullmodell spezifiziert. Dieses ergab für die NK eine Intraklassenkorrelation ICC $=.17$. Danach sind $17 \%$ der beobachteten Leistungsunterschiede in der NK auf Unterschiede zwischen den Schulklassen zurückzuführen, wobei der Varianzanteil zwischen den Klassen auch Leistungsunterschiede zwischen den Schulen enthält. Die übrigen $83 \%$ lassen sich auf Unterschiede zwischen den SuS innerhalb der Schulklassen zurückführen.

Zur Untersuchung der Vorhersage des Kompetenzzuwachses von der 1. zur 3. Klasse wurde in Modell $1 \mathrm{zu}-$ nächst die individuelle Ausgangskompetenz aufgenommen. Erwartungsgemäß stellte sie sich als starker Prädiktor heraus $(\beta=.61, p<.001)$. Die Varianzaufklärung auf Ebene der SuS erreichte durch die Aufnahme der Ausgangskompetenz bereits $38 \%$.

Durch die in Modell 2 auf Individualebene aufgenommenen Familienmerkmale reduzierte sich der Effekt der Ausgangskompetenz auf den Kompetenzzuwachs leicht $(\beta=.55, p<.001)$. Darüber hinaus zeigten sich signifikante Effekte für fast alle Familienmerkmale. So erwiesen sich die Bildung der Eltern $(\beta=.09, p<.001)$, der SES $(\beta=.05$, $p<.001)$, die Anzahl der Bücher $(\beta=.04, p<.001)$ und das Einkommen der Eltern $(\beta=.03, p<.001)$ als bedeutsame Prädiktoren. Die Höhe der Effekte fiel zwar unter Berücksichtigung der Ausgangskompetenz nur sehr gering aus, es lässt sich jedoch erkennen, dass die elterliche Bildung in einem stärkeren Zusammenhang mit der NK steht als soziale Aspekte wie SES oder Einkommen. Ethnischkulturelle Merkmale wie der Migrationshintergrund ( $\beta=$ $-.02, p=.22$ ) oder die zuhause gesprochene Familiensprache $(\beta=.01, p=.63)$ hatten hingegen keinen signifikanten Effekt auf den Zuwachs in der NK. Dafür ergab sich ein kleiner signifikanter Effekt für das Geschlecht $(\beta=-.05, p<$ .001) zugunsten der Jungen. Die individuellen Familienmerkmale erklärten zusammen mit der Ausgangskompetenz in der 1 . Klasse $40 \%$ der Varianz innerhalb der Schulklassen. Damit trugen die Familienmerkmale nur 2\% zur Varianzaufklärung bei.

In Modell 3 zeigte die mittlere Ausgangskompetenz vergleichbar zur Individualebene einen hohen Effekt auf 
die Veränderung der NK $(\beta=.55, p<.001)$. Die Varianzaufklärung auf Klassenebene konnte durch die Hinzunahme des Ausgangsniveaus 30\% erreichen. Dafür sank die Varianzaufklärung der Individualebene wieder auf $38 \%$.

Unter Kontrolle des mittleren Ausgangsniveaus ergab sich in Modell 4 ein signifikanter Effekt für den Anteil an Kindern mit niedrigem SES $(\beta=-.17, p=.03)$ und für den Anteil an Kindern mit mindestens einem Elternteil mit Hochschulabschluss $(\beta=.15, p=.04)$. Für die anderen Kompositionsmerkmale konnten keine signifikanten Effekte nachgewiesen werden. Auf Individualebene reduzierten sich die Effekte der Familienmerkmale geringfügig, dadurch verlor der Prädiktor Einkommen seinen signifikanten Effekt. Insgesamt konnten mit den Kompositionsmerkmalen $12 \%$ der Varianz zwischen den Schulklassen erklärt werden, sodass die Varianzaufklärung für die Klassenebene $42 \%$ betrug.

In Modell 5 zeigte sich, dass die Anzahl an HSU-Stunden einen signifikanten Effekt auf die Veränderung der NK in der 3. Klasse hatte $(\beta=.11, p=.04)$. Der signifikante
Kompositionseffekt des Anteils an Kindern mit niedrigem SES für die NK blieb in diesem Modell bestehen $(\beta=-.17$, $p=.03)$, während der Anteil an Kindern mit Eltern mit Hochschulabschluss nicht mehr signifikant wurde. Das finale Modell zur Vorhersage der NK in der 3. Klasse erreichte auf Individualebene eine Varianzaufklärung von $36 \%$ und auf Klassenebene von $44 \%$.

In Modell 6 ergaben sich sowohl auf Kind- als auch auf Klassenebene indirekte Effekte. Bei den Familienmerkmalen zeigten sich indirekte Effekte für die Anzahl der Bücher $(\beta=.07, p<.001)$, den SES $(\beta=.06, p<.001)$, die Bildung der Eltern $(\beta=.06, p<.001)$ und den Migrationshintergrund $(\beta=-.04, p<.001)$. Vergleichbar zu den direkten Effekten der Familienmerkmale erweisen sich auch die indirekten Effekte als klein. Für die Klassenebene zeigten sich signifikante Effekte für den Anteil an SuS mit Migrationshintergrund $(\beta=-.12, p<.001)$, für den Anteil an SuS mit niedrigem SES $(\beta=-.08, p=.03)$, den Anteil an SuS mit hohem SES $(\beta=.06, p=.03)$ und für die Stundenzahl im Mathematikunterricht $(\beta=.06, p=.01)$.

Tabelle 3. Ergebnisse der Mediationsanalyse zur Vorhersage der naturwissenschaftlichen Kompetenz in Klasse 3

\begin{tabular}{|c|c|c|c|c|}
\hline \multirow{3}{*}{$\begin{array}{l}\text { Variablen } \\
\text { Ebene } 1\end{array}$} & \multicolumn{4}{|c|}{ Modell 6} \\
\hline & \multicolumn{2}{|c|}{ Direkte Effekte } & \multicolumn{2}{|c|}{ Indirekte Effekte } \\
\hline & $\beta$ & $S E$ & $\beta$ & $S E$ \\
\hline Naturwissen. Kompetenz 1. Klasse & $.52^{\star}$ & 0.01 & - & - \\
\hline Geschlecht (1 = männlich) & $-.05^{\star}$ & 0.01 & -.01 & 0.01 \\
\hline Sprache zuhause (1 = deutsch) & .01 & 0.02 & -.01 & 0.01 \\
\hline Migrationshintergrund (1 = kein) & -.02 & 0.02 & $-.04 *$ & 0.01 \\
\hline Anzahl Bücher & $.04^{\star}$ & 0.02 & $.07 \star$ & 0.01 \\
\hline SES (HISEI) & $.05^{\star}$ & 0.02 & $.06^{\star}$ & 0.01 \\
\hline Bildung der Eltern (HISCED) & $.08^{\star}$ & 0.02 & $.06^{\star}$ & 0.01 \\
\hline Einkommen & .03 & 0.02 & .02 & 0.01 \\
\hline \multicolumn{5}{|l|}{ Ebene 2} \\
\hline Mittlere naturwissen. Kompetenz 1. Klasse & $.47^{\star}$ & 0.07 & - & - \\
\hline SuS pro Klasse & -.02 & 0.05 & .01 & 0.02 \\
\hline SuS, männlich (\%) & .02 & 0.05 & .00 & 0.02 \\
\hline SuS, mit Migrationshintergrund (\%) & -.02 & 0.07 & $-.12^{\star}$ & 0.03 \\
\hline SuS, mit niedrigem SES (\%) & $-.17 \star$ & 0.08 & $-.08^{\star}$ & 0.04 \\
\hline SuS, mit hohem SES (\%) & .05 & 0.07 & $.06^{\star}$ & 0.03 \\
\hline SuS, Eltern mit Hochschulabschluss (\%) & .14 & 0.08 & -.02 & 0.03 \\
\hline Unterrichtstunden: Deutsch & .03 & 0.05 & -.01 & 0.02 \\
\hline Unterrichtstunden: Mathematik & .04 & 0.05 & $.06^{\star}$ & 0.02 \\
\hline Unterrichtstunden: HSU & $.11 *$ & 0.05 & .03 & 0.02 \\
\hline
\end{tabular}

Anmerkungen: $N$ = 6,492; $\beta$ = standardisierte Regressionsgewichte, SE = Standardfehler, SES = sozioökonomischer Status, SuS = Schülerinnen und Schüler, $\mathrm{HSU}=$ Heimat - und Sachunterricht.

$* p<.05$. 


\section{Zusammenfassung und Diskussion}

Im vorliegenden Beitrag wurde analysiert, wie sich die NK in der Grundschule entwickelt (Fragestellung 1) und welche Familien- und Klassenmerkmale relevante Effekte auf die Veränderung der NK von der 1. zur 3. Klasse haben (Fragestellung 2 bis 4). Damit sollte zum einen die im Grundschulbereich mangelnde Befundlage zur Entwicklung der NK erweitert und darüber hinaus auch ein Vergleich zu Erkenntnissen über curriculumsbezogene NK (TIMSS, KESS) ermöglicht werden. Zum anderen sollte diese Untersuchung klären, welche Familien- und Klassenmerkmale einen Effekt auf die Veränderung der NK in der Grundschule haben, um mögliche Ansatzpunkte für eine Unterstützung von benachteiligten Personengruppen zu finden. Eine frühe Förderung der NK könnte nicht nur zu einer Verbesserung der NK sowie zu einem höheren Interesse an den Naturwissenschaften führen (Oppermann, Brunner, Eccles \& Anders, 2017), sondern in der Folge auch dazu beitragen, dem Fachkräftemangel in den Natur- und Ingenieurswissenschaften entgegenzuwirken (OECD, 2018).

Zur Untersuchung der Entwicklung der NK über zwei Jahre (Fragestellung 1) haben wir die Effektstärke berechnet, die den Kompetenzzuwachs vergleichbar zu anderen Studien quantifiziert. Dabei zeigte sich für die NK ein $\mathrm{Zu}-$ wachs von $d=1.36$ Standardabweichungen über zwei Schuljahre. Dieser Zuwachs ist mit bestehenden Studien zur frühkindlichen NK vergleichbar (ECLS-K Kindergarten bis Klasse 1: $d=1.59$, ECLS-K Klasse 3 bis $5: d=0.88$, ECLS-K Klasse 5 bis 8: $d=1.14$, Morgan et al., 2016; NEPS Kindergarten bis Klasse 1: $d=1.23$, NEPS Klasse 1 bis 3: $d=1.25$, Kähler, Hahn \& Köller, 2020). Nimmt man eine Gleichverteilung über die Schuljahre an, entspräche dies einem Zuwachs von $d=0.68$ Standardabweichungen pro Schuljahr, der wiederum fast vergleichbar mit dem in der KESS-Studie ermittelten Zuwachs von der 7. zur 8. Klasse ist ( $d=.81$; Ivanov \& Nikolova, 2010). Es ergab sich somit ein Wachstum der NK über die Zeit in der Grundschule, das deutlich über dem in der späten Sekundarstufe I gefundenen lag (TIMSS: $d=.33$, Baumert et al., 1997; PISA: $d=.24$, Walter et al., 2006). Es lassen sich somit auch für die NK nicht-lineare Zuwächse vermuten, d.h. die Leistungszuwächse nehmen in den höheren Klassen immer weiter ab (Köller \& Baumert, 2018). Solche nicht-linearen Verläufe konnten bereits für die mathematische Kompetenz und Lesekompetenz gezeigt werden (Köller \& Baumert, 2018). Allerdings verwenden die hier zum Vergleich herangezogenen Studien wie TIMSS oder KESS curricular valide Aufgaben zur Erfassung der NK, während die NEPS-Naturwissenschaftstests nicht curricular angelegt sind. Es bedarf demnach weiterer Längsschnittuntersuchungen, die die NK an einer ausreichend großen
Stichprobe mit (nicht) curricular angelegten Tests über die gesamte Schulzeit (Primar- und Sekundarstufe) hinweg erheben.

Zur Beantwortung der zweiten bis vierten Fragestellung haben wir in Mehrebenen-Regressionsmodellen die Effekte von Familien- und Klassenmerkmalen auf die NK untersucht. Dabei erwies sich erwartungskonform und vergleichbar zu bestehenden Studien die Ausgangskompetenz in der 1. Klasse als stärkster Prädiktor auf Individualebene, auch unter Berücksichtigung der Familien- und Klassenmerkmale (Baumert et al., 2006; Dumont, Neumann, Nagy et al., 2013; Schiepe-Tiska et al., 2017). Kinder zeigten höhere Kompetenzen in der 3. Klasse, wenn sie bereits in der 1. Klasse eine höhere NK aufwiesen. Dabei konnte allein die Ausgangskompetenz 38\% der Varianz von der NK in der 3. Klasse aufklären. Die Stärke des Effekts verdeutlicht damit auch die Bedeutsamkeit der Erfassung der NK und möglicher Defizite in diesem Bereich, um frühestmöglich eine Förderung zu ermöglichen. Studien konnten hierzu zeigen, dass bereits im Kindergartenalter Disparitäten im Bereich der NK gefunden werden konnten (Hahn \& Schöps, 2019; Kähler, Hahn, Ihme et al., 2020; Kähler, Hahn \& Köller, 2020; Morgan et al., 2016). Diese scheinen sich in der Grundschule fortzusetzen. Um dem Aufbau und der Persistenz dieser Disparitäten entgegenzuwirken, sollte möglichst schon im Kindergartenalter damit begonnen werden, Kinder im Aufbau ihrer NK zu unterstützen. So könnte z.B. ein Curriculum im Kindergarten, das klare Lernziele für die Vorschulzeit formuliert, dabei helfen, spielerisch erstes Wissen über die Naturwissenschaften sowie über Denk- und Arbeitsweisen zu vermitteln. Beispielsweise können sich Kinder bereits im Kindergarten mit Themen wie Aggregatzuständen von Wasser oder dem Lösen und Nicht-Lösen von Stoffen in Wasser auseinandersetzen (Steffensky, Lankes, Carstensen \& Nölke, 2012). Dies könnte der Manifestation von Fehlkonzepten vorbeugen bzw. sie frühzeitig durch wissenschaftliche Vorstellungen ersetzen. Des Weiteren sollte die Grundschule dazu beitragen, Disparitäten zu verringern. Dies bezieht sich vor allem auf den Abbau von naiven Fehlkonzepten oder Alltagsvorstellungen. Dazu muss der Unterricht so gestaltet werden, dass er den Konzeptwechsel und das Lernen naturwissenschaftlich korrekter Vorstellungen begünstigt (Duit \& Häußler, 1997). Der Unterricht sollte den Kindern u.a. die Möglichkeit bieten, eigenständig zu forschen, mit Materialien umzugehen und zu experimentieren (Jonen et al., 2003). Da die Schulstunden in der Grundschule begrenzt sind, könnten naturwissenschaftliche Lernangebote in der Nachmittagsbetreuung angeboten werden, um an einer Reduzierung bestehender Defizite zu arbeiten.

Unter Berücksichtigung der Ausgangskompetenz sollten auch die Effekte bekannter Familienmerkmale untersucht werden. Hier ergaben sich zwar geringe, aber signifi- 
kante Effekte auf das Kompetenzwachstum. Wie erwartet, zeigten Kinder mit höherer elterlicher Bildung, einem höheren SES sowie einer höheren Anzahl an Büchern eine günstigere Veränderung der NK (vgl. Hahn \& Schöps, 2019; Ivanov \& Nikolova, 2010; Wendt et al., 2016). Ein Migrationshintergrund oder eine andere Familiensprache hatten hingegen keinen signifikanten Effekt auf das Wachstum der NK nach Kontrolle anderer Familienmerkmale. Dies kann auf die simultane Berücksichtigung des Ausgangsniveaus zurückgeführt werden. Vergleichbar zu existierenden Studien (vgl. Hahn \& Schöps, 2019), die nach Berücksichtigung sprachlicher Kompetenz keinen Effekt der Familiensprache auf die NK mehr finden konnten, stellt auch in dieser Untersuchung eine Kompetenz (in dem Fall die Ausgangsleistung) einen wichtigeren Prädiktor dar als der ethnisch-kulturelle Hintergrund eines Kindes. Vor allem die Bildung der Eltern scheint eine wichtige Rolle für die NK zu spielen. Unter Berücksichtigung der sozialen Aspekte einer Familie (SES, Einkommen, Anzahl der Bücher) repräsentiert die elterliche Bildung vor allem Kompetenz- und Intelligenzmaße der Eltern. Diese scheinen (wenn auch nur geringfügig) mehr zu der Schulleistungs- und Kompetenzentwicklung der Kinder beizutragen als der soziale Hintergrund der Familie (Lemos, Almeida \& Colom, 2011; Rindermann \& Ceci, 2018). Auch zeigte sich, dass das Einkommen unter Hinzunahme der Kompositionsmerkmale keinen signifikanten Effekt mehr aufwies, während der Effekt der elterlichen Bildung nur geringfügig abnahm. Neben ihrem direkten Effekt wies die Bildung der Eltern auch einen indirekten Effekt auf die NK der 3. Klasse auf. Damit spielt die Bildung der Eltern für die NK bereits am Anfang der Grundschule eine Rolle, welche bis zur 3. Klasse bestehen bleibt. Auch für die Anzahl an Büchern und den SES fanden sich indirekte Effekte. Damit werden soziale Disparitäten auch über die Ausgangskompetenz vermittelt: Kinder mit einem günstigeren familiären Hintergrund weisen gerade dann eine höhere NK in der 3. Klasse auf, wenn vorher ihre NK in der 1. Klasse höher ausfiel. Der Effekt des Migrationshintergrundes wird dabei vollständig über die Ausgangskompetenz vermittelt. Kinder mit Migrationshintergrund wiesen nur dann eine geringere NK in der 3. Klasse auf, wenn ihre NK in der 1. Klasse bereits geringer ausfiel. Auch wenn die Familienmerkmale nur einen geringfügigen Anteil zur Varianzaufklärung der NK in der 3. Klasse beitragen konnten (2\%), wird durch die indirekten Effekte deutlich, dass neben der frühzeitigen Förderung der NK auch der sozialund migrationsbezogene Hintergrund eines Kindes nicht unberücksichtigt bleiben sollte. Zumal bereits im Kindergartenalter sozial- und migrationsbedingte Disparitäten in der NK nachgewiesen werden konnten (Hahn \& Schöps, 2019; Kähler, Hahn, Ihme et al., 2020; Kähler, Hahn \& Köller, 2020; Morgan et al., 2016). Diese scheinen über die Zeit in der Grundschule bestehen zu bleiben. Daher ergibt sich als weiterer Ansatzpunkt zur Verbesserung der NK: Kinder aus sozial schwächeren Familien sollte mehr Unterstützung bei dem Erwerb naturwissenschaftlichen Wissens und dazugehöriger Denk- und Arbeitsweisen geboten werden, um den Rückstand zu ihren Peers über die Schulzeit reduzieren oder gar schließen zu können.

Im Hinblick auf mögliche Geschlechtereffekte zeigten Jungen ein höheres Wachstum an NK als Mädchen. Die Mediationsanalyse offenbarte, dass das Geschlecht jedoch keinen Effekt auf die NK in der 1. Klasse hatte $(\beta=-.01 ; p=.22)$, somit ergab sich auch kein indirekter Effekt des Geschlechts. Geschlechtsbezogene Disparitäten zugunsten der Jungen scheinen sich also erst nach dem 1. Grundschuljahr zu bilden. Studien konnten die Überlegenheit der Jungen im Bereich der Naturwissenschaften bereits umfänglich für die Sekundarstufe I nachweisen: So konnte die KESS-Studie zeigen, dass sich Jungen von der 8. zur 10. Klasse signifikant stärker in der NK verbessern konnten (Ivanov, 2011). Auch der PISA-Längsschnitt 2003/2004 zeigte eine höhere Zunahme der mittleren NK für die Jungen (Walter, Senkbeil, Rost, Carstensen \& Prenzel, 2006). Hier konnte belegt werden, dass Mädchen erst am Ende der 10. Klasse das Kompetenzniveau erreichten, das die Jungen bereits am Ende der 9. Klasse besaßen. Es kann also vermutet werden, dass sich Geschlechtsunterschiede im Lauf der Grundschule bilden. Dabei bleibt unklar, was genau zu dieser Entwicklung führt und wie ihr begegnet werden kann. Hierzu bedarf es weiterer Längsschnittstudien, die die NK in kürzeren Abständen betrachten und gleichzeitig Unterrichtsinhalte und -qualität erfassen.

Neben den individuellen Familienmerkmalen wurden auch verschiedene Klassenmerkmale in den MehrebenenRegressionsanalysen untersucht. Auch auf Klassenebene erwies sich die (mittlere) Ausgangskompetenz der Klasse erwartungskonform als stärkster Prädiktor für die NK in der 3. Klasse (Bellin, 2009; Nikolova, 2011; Schiepe-Tiska et al., 2017). Es zeigt sich also, dass sich nicht nur ein eigenes höheres Ausgangsniveau als relevant für die NK in der 3. Klasse erweist, sondern dass auch das vorangehende Kompetenzniveau in der Klasse eine Rolle spielt. Somit verstärkt sich die bereits weiter oben beschriebene Bedeutsamkeit einer frühen Förderung der NK durch Lernprogramme, Experimente im Unterricht und durch Angebote in der Nachmittagsbetreuung.

Des Weiteren ergab sich ein signifikanter Effekt des Anteils an SuS mit niedrigem SES auf das Kompetenzwachstum. SuS aus Klassen, in denen ein höherer Anteil an Kindern mit niedrigem SES vorlag, zeigten ein geringeres naturwissenschaftliches Wachstum. Damit konnte ein Kompositionseffekt der sozialen Zusammensetzung der Schülerschaft auch für die NK im Grundschulalter nach- 
gewiesen werden (vgl. Baumert et al., 2009; Opdenakker et al., 2002). Insgesamt deuten die Ergebnisse zu der Klassenkomposition darauf hin, dass die soziale Zusammensetzung ebenfalls eine wichtige Determinante für die NK von SuS ist (vgl. Van Ewijk \& Sleegers, 2010). Auch die indirekten Effekte tragen zu dieser Sicht bei: SuS aus Klassen mit einem niedrigeren Anteil an Kindern mit Migrationshintergrund, einem geringeren Anteil an Kindern mit niedrigerem SES oder einem höheren Anteil an Kindern mit hohem SES zeigten in der 3. Klasse dann eine höhere NK auf, wenn ihre NK in der 1. Klasse bereits höher ausfiel. Damit erweist sich die Klassenkomposition bereits zu Beginn der Grundschule als relevantes Merkmal für den $\mathrm{Zu}$ wachs der NK.

Als letzter signifikanter Prädiktor auf Klassenebene hat sich die Anzahl an Stunden im Fach HSU ergeben. Kinder, die mehr HSU erhielten, wiesen damit auch ein höheres Kompetenzwachstum auf. Es bleibt dabei offen, welche Inhalte explizit zu einer Verbesserung des Wachstums von NK beitragen oder inwiefern die Art der Wissensvermittlung (z.B. mittels Experimenten) einen Beitrag zu der Entwicklung von NK leisten kann. Bestehende Studien konnten hierzu den Vorteil von konstruktivistisch orientierten Unterrichtseinheiten im Bereich der Naturwissenschaften aufzeigen, bei denen die Lernenden eine aktive, selbstgesteuerte Rolle einnehmen (Jonen et al., 2003). Demnach muss naturwissenschaftlicher Unterricht Kindern Gelegenheiten bieten, ihre Vorstellungen zu naturwissenschaftlichen Phänomenen (z.B. warum ein Schiff schwimmt) in Gesprächen zu entwickeln, diese in Experimenten zu überprüfen und dazu Erklärungen zu konstruieren. Dabei ist die Auseinandersetzung mit den bestehenden Präkonzepten naturwissenschaftlicher Phänomene besonders wichtig und bedarf einer gezielten Förderung durch strukturierende Elemente. Erst dann kann Unterricht anfänglich naive Fehlkonzepte in wissenschaftlich korrekte Erklärungsmodelle umwandeln (Hardy et al., 2010; Pollmeier et al., 2009).

\section{Limitationen und Ausblick}

Als erste Limitation dieser Studie muss erwähnt werden, dass keine vollständigen Klassen erhoben wurden. Daher musste bzgl. der Kompositionsmerkmale auf Befragungsdaten der Lehrkräfte zurückgegriffen werden, die jedoch fehlerbehaftet sein und von den Angaben der Eltern abweichen können. Auch zeigte sich für die Klassenmerkmale eine deutlich höhere Anzahl an fehlenden Werten, was darauf zurückgeführt werden kann, dass die Lehrkräfte einige Merkmale nur schwer einschätzen konnten oder dies nicht wollten. Da in dieser Erhebung jedoch nur durchschnittlich sechs SuS pro Klasse teilgenommen haben, wären aggregierte Klassenmerkmale deutlich fragwürdiger bzgl. ihrer Aussagekraft gewesen. Es bedarf somit weiterer Studien an vollständigen Klassen, die die gefundenen Effekte auf die Entwicklung der NK bestätigen können.

Des Weiteren sollten zukünftige Studien den Zusammenhang sprachlicher Kompetenzen mit der NK deutlicher untersuchen, da sich hier bereits starke Zusammenhänge ergeben haben (Hahn \& Schöps, 2019; Kähler, Hahn, Ihme et al., 2020). In dieser Studie wurde nur die Familiensprache berücksichtigt, die wiederum nur dichotom (deutsch vs. andere Sprache) erfasst wurde. Daher kann vermutet werden, dass der sprachliche Hintergrund der Kinder nicht ausreichend berücksichtigt wurde. Da Sprache bzw. sprachliche Fähigkeiten jedoch nicht nur eine wichtige Grundlage (schulischen) Lernens darstellen (Kempert, Schalk \& Saalbach, 2019), sondern auch stark mit Umweltfaktoren zusammenhängen (Baumert et al., 2006), kann vermutet werden, dass die gefundenen Effekte der Familien- und Klassenmerkmale überschätzt werden. Es bleibt daher abzuwarten, welche der Familienund Klassenmerkmale sich als Prädiktoren für die NK ergeben, wenn gleichzeitig sprachliche Fähigkeiten berücksichtigt werden.

Auch sollten zukünftige Untersuchungen weitere Aspekte berücksichtigen, die den Kompetenzzuwachs der NK und das Zusammenspiel von Elternhaus und Schule beeinflussen. So blieb in dieser Untersuchung das häusliche Lernumfeld unberücksichtigt, welches nachgewiesenermaßen einen großen Einfluss auf die Kompetenzen junger Kinder hat (Lehrl, 2018). Auch die spezifischen Inhalte des Sachkundeunterrichts wurden im NEPS nicht erfasst und konnten daher nicht berücksichtigt werden. Somit konnten weder Merkmale des häuslichen noch des institutionellen Lernumfelds in dieser Studie einbezogen werden. Es lässt sich vermuten, dass sich die gefundenen Effekte reduzieren würden, wenn gleichzeitig Lerngelegenheiten berücksichtigt werden würden. Daher sollten zukünftige Studien nicht nur mehr Aktivitäten und Lerngelegenheiten sowie deren Inhalte erfassen, es sollten auch indirekte Effekte der familiären Rahmenbedingungen über die Prozessmerkmale betrachtet werden.

Letztendlich liefert die vorliegende Studie erste wertvolle Erkenntnisse über das Zusammenspiel von Familien- und Klassenmerkmalen und dem Wachstum der NK. Weitere Studien, die die Limitationen dieser Untersuchung berücksichtigen, können dazu beitragen, ein genaueres Bild der Wirkmechanismen familiärer und institutioneller Struktur- und Prozessmerkmale auf die frühkindliche NK offen zu legen. All diese Erkenntnisse sollten dann zu einer besseren und gezielten Förderung frühkindlicher NK beitragen. 


\section{Elektronisches Supplement (ESM)}

Das elektronische Supplement ist mit der Online-Version dieses Artikels verfügbar unter https://doi.org/10.1024/ 1010-0652/a000308

ESM 1. NEPS-Rahmenkonzept der Naturwissenschaftstests (PDF)

ESM 2. Beispiel eines NEPS-Items für das naturwissenschaftliche Wissen (PDF)

ESM 3. Beispiel eines NEPS-Items für das Wissen über die Naturwissenschaften (PDF)

ESM 4. Detaillierte Stichprobenbeschreibung (PDF)

ESM 5. Linking-Methode (PDF)

\section{Literatur}

Adams, R. J., Wu, M. L. \& Wilson, M. R. (2015). ACER ConQuest: Generalised item response modelling software (Version 4) [Computer software]. Camberwell, England: Australian Council for Educational Research.

Baumert, J., Lehmann, R., Lehrke, M., Schmitz, B., Clausen, M., Hosenfeld, I. et al. (1997). TIMSS. Mathematisch-naturwissenschaftlicher Unterricht im internationalen Vergleich: Deskriptive Befunde. Opladen: Leske \& Budrich.

Baumert, J., Maaz, K., Stanat, P. \& Watermann, R. (2009). Schulkomposition oder Institution - was zählt? Schulstrukturen und die Entstehung schulformspezifischer Entwicklungsverläufe. Die deutsche Schule, 101, 33-46.

Baumert, J., Stanat, P. \& Watermann, R. (2006). Schulstruktur und die Entstehung differenzieller Lern- und Entwicklungsmilieus. In J. Baumert, P. Stanat \& R. Watermann (Hrsg.), Herkunftsbedingte Disparitäten im Bildungswesen: Differenzielle Bildungsprozesse und Probleme der Verteilungsgerechtigkeit (S. 95 -188). Wiesbaden: VS Verlag für Sozialwissenschaften.

Bellin, N. (2009). Klassenkomposition, Migrationshintergrund und Leistung. Mehrebenenanalysen zum Sprach- und Leseverständnis von Grundschülern. Wiesbaden: VS Verlag für Sozialwissenschaften.

Blossfeld, H.-P., Roßbach, H.-G. \& Maurice, J. v. (2011). Education as a lifelong process: The German National Educational Panel Study (NEPS) [Themenheft]. Zeitschrift für Erziehungswissenschaft, 14. Wiesbaden, Germany: Springer VS.

Bybee, R. W. (1997). Achieving scientific literacy: from purposes to practices. Portsmouth, $\mathrm{NH}$ : Heinemann.

Carey, S. (2009). The origin of concepts. New York: Oxford University Press.

Ditton, H. \& Krüsken, J. (2006). Sozialer Kontext und schulische Leistungen - zur Bildungsrelevanz segregierter Armut. Zeitschrift für Soziologie der Erziehung und Sozialisation, 26 (2), $135-157$.

Duit, R. (1999). Conceptual change approaches in science education. In W. Schnotz, S. Vosniadou \& M. Carretero (Hrsg.), New perspectives on conceptual change (S.263-282). New York: Pergamon.

Duit, R. \& Häußler, P. (1997). Physik und andere naturwissenschaftliche Lernbereiche. In F. E. Weinert (Hrsg.), Psychologie des Unterrichts und der Schule (S.427 - 460). Göttingen: Hogrefe.

Dumont, H., Neumann, M., Maaz, K. \& Trautwein, U. (2013). Die Zusammensetzung der Schülerschaft als Einflussfaktor für Schulleistungen. Psychologie in Erziehung und Unterricht, 60 (3), 163 - 183. https://doi.org/10.2378/peu2013.art14d
Dumont, H., Neumann, M., Nagy, G., Becker, M. \& Rose, N. (2013). Einfluss der Klassenkomposition auf die Leistungsentwicklung in Haupt- und Realschulen in Baden-Württemberg. Psychologie in Erziehung und Unterricht, 60 (3), 198-213. https://doi. org/10.2378/peu2013.art16d

Fischer, L., Rohm, T., Gnambs, T. \& Carstensen, C. (2016). Linking the data of the competence tests (NEPS Survey Paper No. 1). Bamberg, Germany: Leibniz Institute for Educational Trajectories, National Educational Panel Study.

Ganzeboom, H. B. G., de Graaf, P. M. \& Treiman, D. J. (1992). A standard international socio-economic index of occupational status. Social Science Research, 21 (1), 1 -56. https://doi.org/10. 1016/0049-089x(92)90017-B

Gesellschaft für Didaktik des Sachunterrichts. (2002). Perspektivrahmen Sachunterricht. Bad Heilbrunn: Klinkhardt.

Hahn, I. \& Schöps, K. (2019). Bildungsunterschiede von Anfang an? Die Bedeutung von Struktur- und Prozessmerkmalen für die naturwissenschaftliche Kompetenz von Vorschulkindern mit und ohne Migrationshintergrund. Frühe Bildung, 8 (1), 3-12. https://doi.org/10.1026/2191-9186/a000405

Hahn, I., Schöps, K., Rönnebeck, S., Martensen, M., Hansen, S., Saß, S. et al. (2013). Assessing scientific literacy over the lifespan - A description of the NEPS science framework and the test development. Journal for Educational Research Online, 5 (2), 110 - 138.

Hanushek, E. A. \& Wößmann, L. (2015). The knowledge capital of nations: education and the economics of growth. Cambridge, MA: MIT Press.

Hardy, I., Kleickmann, T., Koerber, S., Mayer, D., Möller, K., Pollmeier, J. et al. (2010). Die Modellierung naturwissenschaftlicher Kompetenz im Grundschulalter. Projekt Science-P. In E. Klieme, D. Leutner \& M. Kenk (Hrsg.), Kompetenzmodellierung. Zwischenbilanz des DFG-Schwerpunktprogramms und Perspektiven des Forschungsansatzes (Beiheft der Zeitschrift für Pädagogik, Bd. 56, S.115-125). Weinheim: Beltz.

Hill, C. J., Bloom, H. S., Black, A. R. \& Lipsey, M. W. (2008). Empirical benchmarks for interpreting effect sizes in research. Child Development Perspectives, 2 (3), 172 - 177. https://doi.org/10.1111 /j.1750-8606.2008.00061.x

Ivanov, S. (2011). Naturwissenschaftliche Kompetenz und fachbezogene Einstellungen. In U. Vieluf, S. Ivanov \& R. Nikolova (Hrsg.), KESS 10/11. Kompetenzen und Einstellungen von Schülerinnen und Schülern an Hamburger Schulen am Ende der Sekundarstufe I und zu Beginn der gymnasialen Oberstufe HANSE - Hamburger Schriften zur Qualität im Bildungswesen (Bd. 10, S.183 -214). Münster: Waxmann.

Ivanov, S. \& Nikolova, R. (2010). Naturwissenschaftliche Kompetenzen. In W. Bos \& C. Gröhlich (Hrsg.), KESS 8 - Kompetenzen und Einstellungen von Schülerinnen und Schülern am Ende der Jahrgangsstufe 8 (S.79 - 99). Münster: Waxmann.

Jonen, A., Möller, K. \& Hardy, I. (2003). Lernen als Veränderung von Konzepten - am Beispiel einer Untersuchung zum naturwissenschaftlichen Lernen in der Grundschule. In D. Cech \& H.-J. Schwier (Hrsg.), Lernwege und Aneignungsformen im Sachunterricht (S.93-108). Bad Heilbrunn: Klinkhardt.

Kähler, J. (2019a). NEPS technical report for science: Scaling results of starting cohort 2 for grade 1 (NEPS Survey Paper No. 58). Bamberg, Germany: Leibniz Institute for Educational Trajectories, National Educational Panel Study.

Kähler, J. (2019b). NEPS technical report for science: Scaling results of starting cohort 2 for grade 3 (NEPS Survey Paper No. 60). Bamberg, Germany: Leibniz Institute for Educational Trajectories, National Educational Panel Study.

Kähler, J., Hahn, I., Ihme, J. M. \& Köller, O. (2020). Naturwissenschaftliche Kompetenz von Vorschulkindern. Effekte von Struktur- und Prozessmerkmalen des Elternhauses und der Kindertagesstätteaufdienaturwissenschaftliche Kompetenzvon 4-bis 
6-Jährigen. Psychologie in Erziehung und Unterricht, 67 (OA). https://doi.org/10.2378/peu2020.art29d

Kähler, J., Hahn, I. \& Köller, O. (2020). The development of early scientific literacy gaps in kindergarten children. International Journal of Science Education, 42 (12), 1988 - 2007. https://doi. org/10.1080/09500693.2020.1808908

Kempert, S., Schalk, L. \& Saalbach, H. (2019). Sprache als Werkzeug des Lernens: Ein Überblick zu den kommunikativen und kognitiven Funktionen der Sprache und deren Bedeutung für den fachlichen Wissenserwerb. Psychologie in Erziehung und Unterricht, 66, 176-195. https://doi.org/10.2378/PEU2018. art19d

Köller, O. \& Baumert, J. (2018). Schulische Leistungen und ihre Messung. In W. Schneider \& U. Lindenberger (Hrsg.), Entwicklungspsychologie (8. Aufl., S.663-680). Weinheim: Beltz.

Lehrl, S. (2018). Qualität häuslicher Lernumwelten im Vorschulalter. Eine empirische Analyse zu Konzept, Bedingungen und Bedeutung. Wiesbaden: Springer VS.

Lemos, G. C., Almeida, L. S. \& Colom, R. (2011). Intelligence of adolescents is related to their parents' educational level but not to family income. Personality and Individual Differences, 50 (7), 1062 - 1067. https://doi.org/10.1016/j.paid.2011.01.025

Lüdtke, O., Robitzsch, A. \& Grund, S. (2017). Multiple imputation of missing data in multilevel designs. A comparison of different strategies. Psychological Methods, 22 (1), $141-165$. https://doi. org/10.1037/met0000096

Morgan, P. L., Farkas, G., Hillemeier, M. M. \& Maczuga, S. (2016). Science achievement gaps begin very early, persist, and are largely explained by modifiable factors. Educational Researcher, 45 (1), 18 - 35. https://doi.org/10.3102/0013189X16633182

Muthén, L. K. \& Muthén, B. O. (2012). Mplus (Version 7.4) [Computer software]. Los Angeles, CA: Muthén \& Muthén.

Niklas, F., Segerer, R., Schmiedeler, S. \& Schneider, W. (2012). Findet sich ein "Matthäus-Effekt“ in der Kompetenzentwicklung von jungen Kindern mit oder ohne Migrationshintergrund? Frühe Bildung, 1 (1), 26 - 33. https://doi.org/10.1026/2191-9186/a000022

Nikolova, R. (2011). Grundschulen als differenzielle Entwicklungsmilieus. Objektive Kontextmerkmale der Schülerzusammensetzung und deren Auswirkungen auf die Mathematik- und Leseleistungen. Münster: Waxmann.

OECD. (2006). Assessing scientific, reading and mathematical literacy: A framework for PISA 2006. Paris, France: OECD Publishing.

OECD. (2018). PISA for development assessment and analytical framework: Reading, mathematics and science. Paris, France: OECD Publishing.

Opdenakker, M.-C., van Damme, J., Fraine, B. de, van Landeghem, G. \& Onghena, P. (2002). The effect of schools and classes on mathematics achievement. School Effectiveness and School Improvement, 13 (4), 399-427. https://doi.org/10.1076/ sesi.13.4.399.10283

Oppermann, E., Brunner, M., Eccles, J. S. \& Anders, Y. (2017). Uncovering young children's motivational beliefs about learning science. Journal of Research in Science Teaching, 55 (3), 399 - 421. https://doi.org/10.1002/tea.21424

Pollmeier, J., Kleickmann, T., Hardy, I., Tröbst, S., Möller, K. \& Schwippert, K. (2009). Entwicklung naturwissenschaftlicher Kompetenz in der Grundschule (Science-P): Naturwissenschaftliches Wissen. In C. Röhner, C. Henrichwark \& M. Hopf (Hrsg.), Europäisierung der Bildung (S. 199 - 203). Wiesbaden:VS Verlag für Sozialwissenschaften.

Rindermann, H. \& Ceci, S. J. (2018). Parents' education is more important than their wealth in shaping their children's intelligence. Results of 19 samples in seven countries at different developmental levels. Journal for the Education of the Gifted, 41 (4), 298 - 326. https://doi.org/10.1177/0162353218799481
RStudio Team (2015). RStudio: Integrated development for R (Version 3.5.2) [Computer software]. Boston, MA: RStudio, Inc.

Schiepe-Tiska, A., Rönnebeck, S., Heitmann, P., Schöps, K., Prenzel, M. \& Nagy, G. (2017). Die Veränderung der naturwissenschaftlichen Kompetenz von der 9. zur 10. Klasse bei PISA und den Bildungsstandards unter Berücksichtigung geschlechts- und schulartspezifischer Unterschiede sowie der Zusammensetzung der Schülerschaft. Zeitschrift für Erziehungswissenschaft, 20 (S2), 151 - 176. https://doi.org/10.1007/s11618-017-0754-2

Schroedter, J. H., Lechert, Y. \& Lüttinger, P. (2006). ZUMA-Methodenbericht 2006/08. Die Umsetzung der Bildungsskala ISCED-1997 für die Volkszählung 1970, die Mikrozensus-Zusatzerhebung 1971 und die Mikrozensen 1976 - 2004 (Version 1).

Schwippert, K., Wendt, H. \& Tarelli, I. (2012). Lesekompetenzen von Schülerinnen und Schülern mit Migrationshintergrund. In W. Bos, I. Tarelli, A. Bremerich-Vos \& K. Schwippert (Hrsg.), IGLU 2011. Lesekompetenzen von Grundschulkindern in Deutschland im internationalen Vergleich (S.191-207). Münster: Waxmann.

Sodian, B., Jonen, A., Thoermer, C. \& Kircher, E. (2006). Die Natur der Naturwissenschaften verstehen - Implementierung wissenschaftstheoretischen Unterrichts in der Grundschule. In M. Prenzel \& J. Allolio-Näcke (Hrsg.), Untersuchungen zur Bildungsqualität von Schule. Abschlussbericht des DFG-Schwerpunktprogramms (S.147-160). Münster: Waxmann.

Sodian, B. \& Mayer, D. (2013). Entwicklung des wissenschaftlichen Denkens im Vor- und Grundschulalter. In M. Stamm \& D. Edelmann (Hrsg.), Handbuch frühkindliche Bildungsforschung (S.617). Wiesbaden: Springer Fachmedien.

Steffensky, M., Lankes, E.-M., Carstensen, C. H. \& Nölke, C. (2012). Alltagssituationen und Experimente: Was sind geeignete naturwissenschaftliche Lerngelegenheiten für Kindergartenkinder? Ergebnisse aus dem SNaKE-Projekt. Zeitschrift für Erziehungswissenschaft, 15 (1), $37-54$. https://doi.org/10.1007/s11618012-0262-3

Van Ewijk, R. \& Sleegers, P. (2010). The effect of peer socioeconomic status on student achievement. A meta-analysis. Educational Research Review, 5 (2), 134-150. https://doi.org/10. 1016/j.edurev.2010.02.001

Walter, O. (2008). Ethno-linguale Kompositionseffekte in neunten Klassen: Befunde aus der Klassenstichprobe von PISA 2006. Zeitschrift für Erziehungswissenschaft, 10, 169-184.

Walter, O., Senkbeil, M., Rost, J., Carstensen, C. \& Prenzel, M. (2006). Die Entwicklung der naturwissenschaftlichen Kompetenz von der neunten zur zehnten Klassenstufe: Deskriptive Befunde. In M. Prenzel, J. Baumert, W. Blum, R. Lehmann, D. Leutner, M. Neubrand et al. (Hrsg.), PISA 2003. Untersuchungen zur Kompetenzentwicklung im Verlauf eines Schuljahres (S.88-118). Münster: Waxmann.

Wendt, H., Bos, W., Selter, C., Köller, O., Schwippert, K. \& Kasper, D. (2016). TIMSS 2015. Mathematische und naturwissenschaftliche Kompetenzen von Grundschulkindern in Deutschland im internationalen Vergleich. Münster: Waxmann.

Zimmerman, C. (2007). The development of scientific thinking skills in elementary and middle school. Developmental Review, 27 (2), 172 - 223. https://doi.org/10.1016/j.dr.2006.12.001

\section{Historie}

Manuskript eingereicht: 03.06.2020

Manuskript nach Revision angenommen: 01.02.2021

Onlineveröffentlichung: 12.02.2021

\section{Förderung}

Open Access-Veröffentlichung ermöglicht durch das IPN Leibniz-Institut für die Pädagogik der Naturwissenschaften und Mathematik, Kiel. 
ORCID

Jana Kähler

(D) https://orcid.org/0000-0002-2694-4531

\section{Jana Kähler}

IPN Leibniz-Institut für die Pädagogik

der Naturwissenschaften und Mathematik

Olshausenstraße 62

$24118 \mathrm{Kiel}$

Deutschland

jkaehler@leibniz-ipn.de 